Correspondence

Michael G. Surette surette@ucalgary.ca

Received 9 October 2009 Accepted 20 January 2010

\section{McKay agar enables routine quantification of the 'Streptococcus milleri' group in cystic fibrosis patients}

\author{
Christopher D. Sibley, ${ }^{1}$ Margot E. Grinwis, ${ }^{1}$ Tyler R. Field, ${ }^{1}$ \\ Michael D. Parkins, ${ }^{2,3}$ Jens C. Norgaard, ${ }^{1}$ Daniel B. Gregson, ${ }^{2,4,5}$
} Harvey R. Rabin ${ }^{1,2,3}$ and Michael G. Surette ${ }^{1,6}$

${ }^{1}$ Department of Microbiology and Infectious Diseases, University of Calgary, Calgary, AB T2N 4N1, Canada

${ }^{2}$ Department of Medicine, University of Calgary, Calgary, AB T2N 4N1, Canada

${ }^{3}$ Adult Cystic Fibrosis Clinic, University of Calgary, Calgary, AB T2N 4N1, Canada

${ }^{4}$ Division of Microbiology, Calgary Laboratory Services No. 9, 3535 Research Road NW, Calgary, AB T2L 2K8, Canada

${ }^{5}$ Department of Pathology and Laboratory Medicine, University of Calgary, 3330 Hospital Drive NW, Calgary, AB T2N 4N1, Canada

${ }^{6}$ Department of Biochemistry and Molecular Biology, University of Calgary, Calgary, AB T2N 4N1, Canada

\begin{abstract}
The 'Streptococcus milleri' group (SMG) has recently been recognized as a contributor to bronchopulmonary disease in cystic fibrosis (CF). Routine detection and quantification is limited by current CF microbiology protocols. McKay agar was developed previously for the semi-selective isolation of this group. Here, McKay agar was validated against a panel of clinical SMG isolates, which revealed improved SMG recovery compared with Columbia blood agar. The effectiveness of this medium was evaluated by appending it to the standard CF sputum microbiology protocols in a clinical laboratory for a 6-month period. All unique colony types were isolated and identified by $16 \mathrm{~S}$ rRNA gene sequencing. Whilst a wide variety of organisms were isolated, members of the SMG were the most prevalent bacteria cultured, and McKay agar allowed routine quantification of the SMG from $10^{3}$ to $>10^{8}$ c.f.u. $\mathrm{ml}^{-1}$ directly from sputum. All members of the SMG were detected [Streptococcus anginosus (40.7\%), Streptococcus intermedius (34.3\%) and Streptococcus constellatus (25\%)] with an overall prevalence rate of $40.6 \%$ in our adult CF population. Without exception, samples where SMG isolates were cultured at $10^{7}$ c.f.u. $\mathrm{ml}^{-1}$ or greater were associated with pulmonary exacerbations. This study demonstrates that McKay agar can be used routinely to quantify the SMG from complex clinical samples.
\end{abstract}

\section{INTRODUCTION}

The 'Streptococcus milleri' group (SMG), also referred to as the Streptococcus anginosus group, comprises Streptococcus anginosus, Streptococcus constellatus and Streptococcus intermedius. SMG isolates are members of the healthy human microbiota and asymptomatically colonize the mouth, nasopharynx, gastrointestinal tract and genitourinary tract of $15-40 \%$ of the general population (Poole \& Wilson, 1979). However, the SMG has a propensity to cause severe purulent infections at virtually all body sites.

Abbreviations: CF, cystic fibrosis; OF, oropharyngeal flora; SMG, 'Streptococcus milleri' group.
Notably, members of the SMG are the most common cause of brain and liver abscesses and pulmonary empyema (Maskell et al., 2005; Moore-Gillon et al., 1981; Prasad et al., 2006). Furthermore, the SMG has been associated with as much invasive disease as all other pyogenic streptococci combined (Laupland et al., 2006). The SMG as a cause of non-invasive disease has only rarely been assessed. However, data suggest that this group may be responsible for a large burden of 'culture-negative' community-acquired pneumonia (Ishida et al., 1998; Shinzato \& Saito, 1995; Whiley et al., 1992).

Cystic fibrosis (CF) is the most common lethal genetic disease in the white population. Chronically progressive 
bouts of endobronchial infection and inflammation ultimately lead to death in $90 \%$ of afflicted individuals (Lyczak et al., 2002). CF microbiology protocols have been developed for the specific isolation of a number of chronically colonizing/infecting organisms associated with chronic CF airway infection, including Pseudomonas aeruginosa, Staphylococcus aureus, the Burkholderia cepacia complex and more recently Aspergillus spp., Stenotrophomonas maltophilia and Achromobacter xylosoxidans (Gilligan, 1991; Miller \& Gilligan, 2003). However, culture-independent molecular approaches suggest that CF airway disease has a much more complex polymicrobial aetiology, whereby complex and dynamic microbial community interactions may lead to enhanced pathogenesis (Bittar et al., 2008; Harris et al., 2007; Rogers et al., 2003, 2004, 2005, 2006; Sibley et al., 2006, 2008a, b). Recently, we implicated the SMG as clinically relevant members of the CF lung microbiome because of their association with pulmonary exacerbations (Parkins et al., 2008a, b; Sibley et al., 2008b).

Standard microbiological practices recommended for $\mathrm{CF}$ have overlooked the clinical significance of the SMG because they are frequently underrepresented on the solid media recommended for CF microbiology. Additionally, SMG colonies are phenotypically indistinguishable from those of other streptococci, which traditionally have been considered to have low or no pathogenic potential. To overcome this limitation, we previously developed McKay agar, a complex semi-selective solid medium (Sibley et al., 2008b). In this study, we evaluated McKay agar for the isolation and quantification of the SMG in CF sputum by prospectively surveying an adult CF cohort for 6 months, by adding it to the standard CF sputum microbiology protocols in a clinical laboratory.

\section{METHODS}

McKay agar. One litre of McKay agar is made by combining the following and adjusting the $\mathrm{pH}$ to 7.2 before autoclave sterilization: $13.3 \mathrm{~g}$ nutrient broth (BD), 5 g glucose, 10 g yeast extract (Difco), $5 \mathrm{~g}$ tryptone (BD), $2 \mathrm{~g} \mathrm{~K}_{2} \mathrm{HPO}_{4}, 40 \mathrm{ml}$ salt solution $\left(10 \mathrm{~g} \mathrm{NaHCO}_{3} \mathrm{l}^{-1}\right.$, $2 \mathrm{~g} \mathrm{NaCl} \mathrm{l}^{-1}, 1 \mathrm{~g} \mathrm{~K}_{2} \mathrm{HPO}_{4} \mathrm{l}^{-1}, 1 \mathrm{~g} \mathrm{KH}_{2} \mathrm{PO}_{4} \mathrm{l}^{-1}, 0.5 \mathrm{~g} \mathrm{MgSO}_{4} .7 \mathrm{H}_{2} \mathrm{O}$ $\left.\mathrm{l}^{-1}, 0.25 \mathrm{~g} \mathrm{CaCl}_{2} .2 \mathrm{H}_{2} \mathrm{O} \mathrm{l}^{-1}\right), 1 \mathrm{ml}$ Tween $80,1 \mathrm{mg}$ crystal violet, $60 \mathrm{mg}$ bromocresol purple, $10 \mu \mathrm{g}$ vitamin $\mathrm{K}, 0.005 \mathrm{~g}$ haemin and $15 \mathrm{~g}$ Bacto agar (Difco) (Sibley et al., 2008b). The sterilized medium is supplemented with $20 \mathrm{ml} \mathrm{L}$-arginine $(2.5 \%, \mathrm{w} / \mathrm{v})$ and filtersterilized antibiotic stocks: sulfadiazine $\left(16 \mathrm{mg} \mathrm{ml}^{-1}\right.$ in $0.1 \mathrm{M}$ $\mathrm{NaOH})$, colistin sulfate $\left(1 \mathrm{mg} \mathrm{ml} \mathrm{m}^{-1}\right.$ in $\left.\mathrm{dH}_{2} \mathrm{O}\right)$ and oxolinic acid $\left(0.5 \mathrm{mg} \mathrm{ml}^{-1}\right.$ in $\left.0.1 \mathrm{M} \mathrm{NaOH}\right)$ to final concentrations of 500,10 and $5 \mu \mathrm{g} \mathrm{ml}^{-1}$, respectively.

Sample collection and processing. To evaluate the utility of McKay agar in a clinical laboratory, we prospectively surveyed an adult CF cohort for a 6-month period (mid-September 2007 to midMarch 2008) by appending McKay agar culture to the standard protocol for CF microbiology in a clinical laboratory. Sputum specimens were collected in accordance with ethical guidelines during routine patient care. An equal volume of dithiothreitol $\left(10 \mathrm{mg} \mathrm{ml}^{-1}\right)$ was mixed with the sputum by vortexing, followed by incubation at room temperature for at least $15 \mathrm{~min}$. Quantitative analysis was carried out by serial dilution; the $10^{-3}$ and $10^{-5}$ dilutions were plated on solid media including Columbia blood agar (CBA), chocolate agar, MacConkey agar, mannitol-salt agar, oxidation-fermentation polymyxin bacitracin lactose agar (OFPBL) and McKay agar. Plates were incubated at $35{ }^{\circ} \mathrm{C}$ in the presence of $5 \% \mathrm{CO}_{2}$ for 2 days, except for the McKay agar culture, which was incubated for 4 days, the OFPBL culture, which was incubated at $30{ }^{\circ} \mathrm{C}$, and the chocolate agar culture, which was incubated under anaerobic conditions. All differential colony types were quantified and colony morphologies were documented daily. Isolates cultivated on McKay agar were purified twice by McKay agar passage before scoring their ability to change the medium to a yellow colour.

To measure the recovery efficiencies of CBA and McKay agar, CF and non-CF SMG isolates were grown overnight in brain-heart infusion (BHI) broth, serially diluted in $0.85 \% \mathrm{NaCl}$, and $5 \mu \mathrm{l}$ of each dilution (in triplicate) was cultivated on McKay, CBA and BHI agars. The ratio of c.f.u. recovered on CBA versus McKay agar was used to determine the recovery efficiency.

Bacterial identification. Bacterial strains were identified by PCR amplification and sequencing of part of the 16S rRNA gene using primers 8f and 926r (Liu et al., 1997). Identification to the species level corresponds to $>97 \%$ identity to the closest match in the RDP database (http://rdp.cme.msu.edu/). The 16S rRNA gene is a reliable target for SMG speciation (Daley et al., 2005).

\section{RESULTS AND DISCUSSION}

\section{McKay agar has superior SMG recovery efficiencies to CBA}

McKay agar was developed as a semi-selective solid medium for the isolation of the SMG from non-sterile body sites such as CF airways (Sibley et al., 2008b). The growth medium is supplemented with the antibiotics colistin and oxolinic acid to inhibit the growth of the principal CF pathogens (P. aeruginosa and Staphylococcus aureus). It is supplemented with sulfadiazine to select for the SMG and against the bulk of other viridans streptococci (Brogan et al., 1997; Cooke \& O’Neill, 1998; Poole \& Wilson, 1979). Bromocresol purple is included as a colorimetric $\mathrm{pH}$ indicator to identify those isolates that produce an acidic environment on this medium, a further distinguishing feature of SMG colonies.

Initial studies have suggested that a proportion of SMG isolates might evade detection by routine CF microbiology protocols due to a failure to be recovered efficiently on CBA (Sibley et al., 2008b). To specifically address this, we validated McKay agar against a panel of 22 SMG clinical isolates (11 non-CF isolates and $11 \mathrm{CF}$ isolates). The ratio of colonies detected on CBA versus McKay agar revealed that eight of the 22 strains tested were dramatically underrepresented on CBA (Fig. 1a). Improved McKay agar recovery was noted for all three species (Streptococcus anginosus, Streptococcus intermedius and Streptococcus constellatus). For example, CBA underrepresented the Streptococcus constellatus strain C88 by $10^{5}$-fold relative to McKay agar, whereas for strain C245, CBA and McKay agar had equal recovery efficiencies. Three representative examples of SMG strains with differential recovery on 

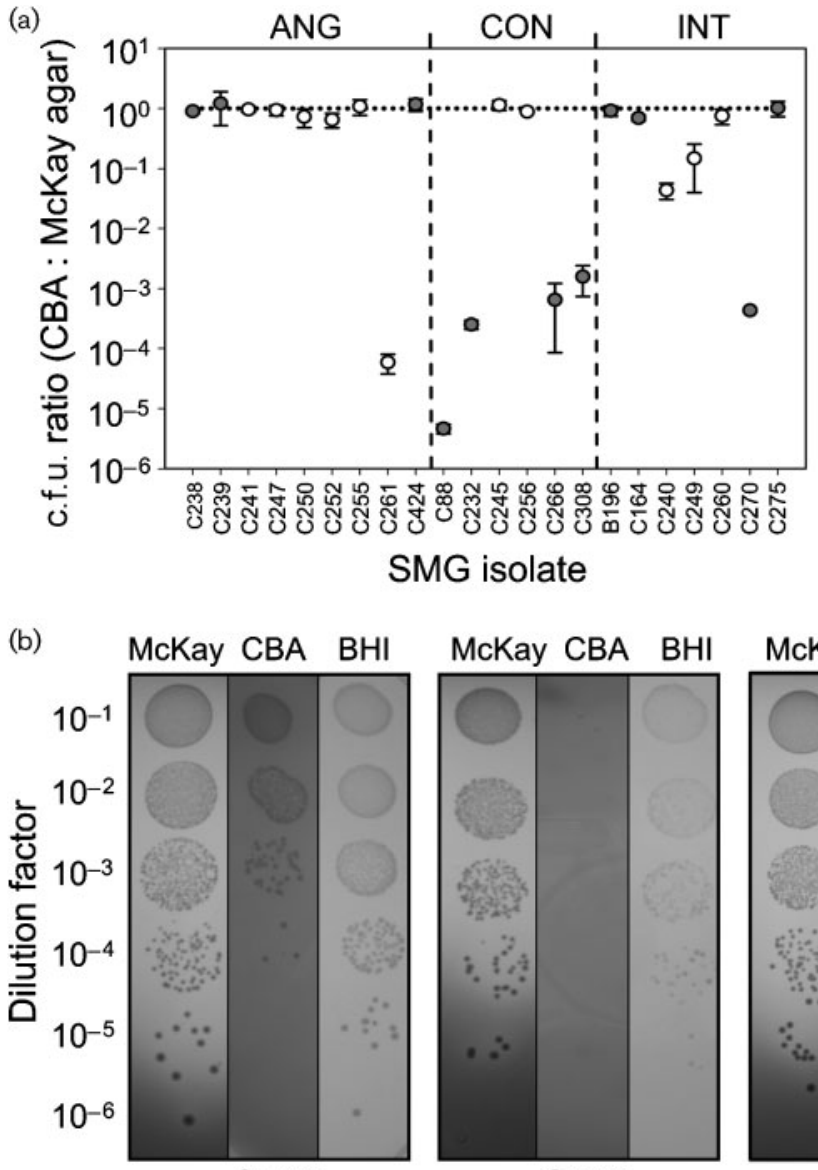

$\mathrm{C} 240$

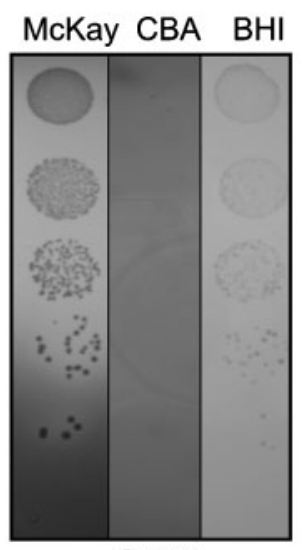

$\mathrm{C} 270$

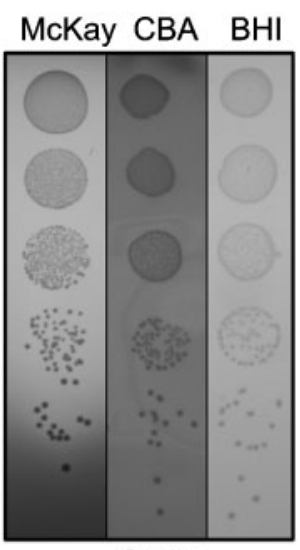

C245
Fig. 1. (a) McKay agar is superior to CBA at recovering the SMG. Clinical isolates [representing invasive isolates (open circles) and CF isolates (shaded circles)] were grown overnight in $\mathrm{BHI}$ broth, serially diluted in saline and plated onto McKay agar, CBA and $\mathrm{BH}$ agar. The ratio of SMG colonies present on CBA:McKay agar is plotted. ANG, Streptococcus anginosus; CON, Streptococcus constellatus; INT, Streptococcus intermedius. (b) Examples of SMG strains with reduced recovery on CBA (isolates C240 and C270) and an example of a strain with equal recovery on both McKay agar and CBA (isolate C245). The recovery of the SMG on McKay and $\mathrm{BHI}$ agar was equal for all strains tested.
CBA and McKay agar are shown in Fig. 1(b). All SMG strains tested were cultured in equal proportions on $\mathrm{BHI}$ and McKay agar (data not shown). These results showed that, even when plated from robust overnight cultures, viable numbers of SMG can be significantly underestimated on CBA.

\section{McKay agar culture surveillance of an adult CF population}

During the 6 months that we surveyed the adult CF cohort, we were able to screen $106 / 128$ patients $(83 \%)$ in the clinic population ( $45.3 \%$ female, $54.7 \%$ male) with McKay agar culture. This provided 226 samples (mean of 2.1 sputum samples per patient) comprising 180 routine surveillance samples and 46 samples collected during bronchopulmonary exacerbation.

All visually distinct colony morphologies cultured on McKay agar were isolated and purified twice by serial passage. In total, 813 isolates were documented on the initial growth plates; however, 115 failed to grow upon purification. A mean of 3.1 morphologically distinct colony types were identified from each sputum culture on McKay agar. 16S rRNA gene PCR products for 647/698 (93\%) of the isolates yielded high-quality DNA suitable for analysis.
Fifty distinct bacterial species were identified throughout the study (Table 1). The distribution of these isolates in the patient population is shown in Table 1. The recovery of known CF pathogens was uncommon. Twenty-two isolates $(3.4 \%)$ represented traditionally recognized CF pathogens and comprised Staphylococcus aureus (13 isolates), Burkholderia cenocepacia (three), Streptococcus pneumoniae (three), Burkholderia multivorans (two) and Inquilinus limosus (one).

In total, $108 \mathrm{SMG}$ strains were isolated (16.7\% of the total isolates collected) from 75 of the 226 sputum specimens (33.2\%). SMG strains were recovered from 43 patients (30 males and 13 females), representing a prevalence rate of $40.6 \%$ in the assessed adult CF population. All three species of the SMG were detected, with Streptococcus anginosus being the most common ( $41 \%$ of SMG), followed by Streptococcus intermedius (34\%) and Streptococcus constellatus (25\%). Streptococcus anginosus was isolated from 18 males and six females, Streptococcus intermedius from 11 males and 11 females, and Streptococcus constellatus from ten males and one female. Twelve sputum samples, from nine patients, co-cultured multiple SMG species. The most common association was Streptococcus anginosus and Streptococcus constellatus (8/12), followed by Streptococcus anginosus and Strepto- 
Table 1. Characteristics of isolates collected on McKay agar

\begin{tabular}{|c|c|c|c|c|}
\hline 16S rRNA classification & $\begin{array}{l}\text { No. }(\%) \text { of times isolated } \\
\qquad(n=647)\end{array}$ & $\begin{array}{l}\text { No. (\%) of patients } \\
\qquad(n=106)\end{array}$ & c.f.u. range & $\begin{array}{l}\text { No. }(\%) \text { that changed } \\
\text { media indicator }\end{array}$ \\
\hline Streptococcus salivarius & $149(23)$ & $77(72.6)$ & $10^{3}-10^{7}$ & $140(94)$ \\
\hline Streptococcus anginosus & $44(6.8)$ & $24(22.6)$ & $10^{3}-10^{6}$ & $41(93.2)$ \\
\hline Streptococcus intermedius & $37(5.7)$ & $22(20.8)$ & $10^{3}-10^{8}$ & $34(91.9)$ \\
\hline Streptococcus sp.* & $59(9.1)$ & $37(34.9)$ & $10^{3}-10^{8}$ & $23(39.0)$ \\
\hline Gemella haemolysans & $53(8.1)$ & $30(28.3)$ & $10^{3}-10^{6}$ & $37(69.8)$ \\
\hline Streptococcus sanguinis & $43(6.6)$ & $25(23.6)$ & $10^{4}-10^{6}$ & $32(74.4)$ \\
\hline Streptococcus pneumoniae/mitis $\dagger$ & $39(6.0)$ & $33(31.1)$ & $10^{4}-10^{6}$ & $1(2.6)$ \\
\hline Gemella sanguinis & $20(3.1)$ & $16(15.1)$ & $10^{3}-10^{6}$ & $11(55.0)$ \\
\hline Streptococcus mitis & $19(2.9)$ & $18(16.9)$ & $10^{3}-10^{7}$ & $0(0)$ \\
\hline Streptococcus parasanguinis & $9(1.4)$ & $7(6.6)$ & $10^{3}-10^{6}$ & $0(0)$ \\
\hline Streptococcus mutans & $8(1.2)$ & $8(7.5)$ & $10^{3}-10^{5}$ & $8(100)$ \\
\hline Streptococcus oralis & $8(1.2)$ & $8(7.5)$ & $10^{4}-10^{7}$ & $8(100)$ \\
\hline Rothia mucilaginosa & $7(1.1)$ & $4(3.8)$ & $10^{3}-10^{5}$ & $7(100)$ \\
\hline Granulicatella sp.* & $5(0.8)$ & $5(4.7)$ & $10^{3}-10^{5}$ & $3(60.0)$ \\
\hline Lactobacillus casei & $5(0.8)$ & $3(2.8)$ & $10^{3}-10^{5}$ & $5(100)$ \\
\hline Streptococcus gordonii & $5(0.8)$ & $5(4.7)$ & $10^{5}$ & $1(20.0)$ \\
\hline Gemella morbillorum & $4(0.6)$ & $2(1.9)$ & $10^{5}$ & $1(25.0)$ \\
\hline Granulicatella adiacens & $4(0.6)$ & $3(2.8)$ & $10^{5}-10^{6}$ & $1(25.0)$ \\
\hline Streptococcus infantis & $4(0.6)$ & $2(1.9)$ & $10^{3}-10^{6}$ & $4(100)$ \\
\hline Streptococcus pyogenes & $4(0.6)$ & $1(0.9)$ & $10^{6}-10^{7}$ & $4(100)$ \\
\hline Burkholderia multivorans & $2(0.3)$ & $1(0.9)$ & $10^{3}$ & $0(0)$ \\
\hline Enterococcus raffinosus & $2(0.3)$ & $1(0.9)$ & $10^{6}-10^{7}$ & $2(100)$ \\
\hline Lactobacillus acidophilus & $2(0.3)$ & $1(0.9)$ & $10^{3}-10^{4}$ & $2(100)$ \\
\hline Lactobacillus gasseri & $2(0.3)$ & $2(1.9)$ & $10^{3}-10^{5}$ & $2(100)$ \\
\hline Streptococcus agalactiae & $2(0.3)$ & $2(1.9)$ & $10^{4}-10^{5}$ & $2(100)$ \\
\hline Streptococcus australis & $2(0.3)$ & $2(1.9)$ & $10^{5}$ & $1(50)$ \\
\hline Actinomyces naeslundii & $1(0.2)$ & $1(0.9)$ & $10^{5}$ & $1(100)$ \\
\hline Enterococcus faecalis & $1(0.2)$ & $1(0.9)$ & $10^{3}$ & $1(100)$ \\
\hline Enterococcus faecium & $1(0.2)$ & $1(0.9)$ & $10^{6}$ & $0(0)$ \\
\hline Inquilinus limosus & $1(0.2)$ & $1(0.9)$ & $10^{5}$ & $0(0)$ \\
\hline Lactobacillus paracasei & $1(0.2)$ & $1(0.9)$ & $10^{4}$ & $1(100)$ \\
\hline Lactobacillus salivarius & $1(0.2)$ & $1(0.9)$ & $10^{3}$ & $1(100)$ \\
\hline Lactobacillus sp.* & $1(0.2)$ & $1(0.9)$ & $10^{5}$ & $1(100)$ \\
\hline Micrococcus luteus & $1(0.2)$ & $1(0.9)$ & $10^{3}$ & $0(0)$ \\
\hline Rothia dentocariosa & $1(0.2)$ & $1(0.9)$ & $10^{6}$ & $0(0)$ \\
\hline Streptococcus cristatus & $1(0.2)$ & $1(0.9)$ & $10^{4}$ & $0(0)$ \\
\hline Streptococcus massiliensis & $1(0.2)$ & $1(0.9)$ & $10^{5}$ & $1(100)$ \\
\hline Streptococcus vestibularis & $1(0.2)$ & $1(0.9)$ & $10^{5}$ & $1(100)$ \\
\hline
\end{tabular}

${ }^{\star}$ Isolates that could only be assigned to the genus level based on $16 \mathrm{~S}$ rRNA gene sequence. It is possible that these groups represent more than one bacterial species.

$\dagger$ Identity could not be discriminated between Streptococcus pneumoniae and Streptococcus mitis based on partial 16S rRNA gene sequence. 
coccus intermedius (3/12). Streptococcus intermedius and Streptococcus constellatus were co-cultivated from a single sputum sample.

The prevalence of co-colonizing pathogens in SMGpositive specimens identified using traditional CF protocols was: P. aeruginosa, $73 \%$; Staphylococcus aureus, $28.6 \%$ (meticillin-sensitive $S$. aureus $25.4 \%$, meticillin-resistant $S$. aureus $3.2 \%$ ); Streptococcus pneumoniae, $1.6 \%$; Stenotrophomonas maltophilia, $1.6 \%$; Streptococcus pyogenes, $1.6 \%$; and I. limosus, $1.6 \%$. This was not significantly different from the normal distribution of these pathogens in our CF population (data not shown).

Reported SMG carriage rates vary depending on the body site that is sampled. For example, carriage rates in the vagina are $18.2 \%$, whereas SMG bacteria are cultured from $31 \%$ of throat swabs if sulfonamide selection is employed (as with McKay agar) (Poole \& Wilson, 1979). Poole \& Wilson (1979) showed that SMG carriage rates increased with age in the nasopharynx from $0 \%$ in individuals $<5$ years old to $25 \%$ in individuals aged 5-14, and up to $43 \%$ in individuals aged $>15$ years. Our carriage rate was consistent with these findings, given that all patients screened in this study were adults ( $>18$ years).

\section{Evaluation of McKay agar}

As indicated in Table 1 , a wide variety of organisms were recovered from sputum at $37{ }^{\circ} \mathrm{C}$ in the presence of $5 \%$ $\mathrm{CO}_{2}$ using McKay agar culture. However, the value of culturing is not just in determining the presence or absence of an organism but also in accurately measuring abundance. Notably, members of the SMG were the most abundant organisms detected by McKay agar representing more than half of the total c.f.u. recovered (Fig. 2). It is unclear how the concentration of obligately anaerobic airway flora would have altered these data because anaerobic McKay agar culture was not carried out. Throughout the study, the SMG was recovered from eight patients on at least three separate occasions at concentrations above $10^{6}$ c.f.u. $\mathrm{ml}^{-1}$, suggesting high-level chronic colonization in these individuals.

The majority of the bacterial isolates recovered on McKay agar were members of the viridans group streptococci (457 isolates; $65.5 \%$ of the isolates collected), represented by at least 18 different species (Table 1). Although Streptococcus salivarius was the most common bacterial species to be recovered [149 isolates from 77 patients $(72.6 \%$ of the population)], it only represented $11.2 \%$ of the total c.f.u. recovered (Fig. 2). We recovered 59 isolates that could not be assigned to a species using the partial $16 \mathrm{~S}$ rRNA gene sequence but were assigned to the genus Streptococcus ( $<97 \%$ identical to known Streptococcus species). Collectively, these organisms represented the second most abundant number of c.f.u. to be recovered on McKay agar. Genera that also included members not assigned to a species included Gemella (ten isolates), Granulicatella

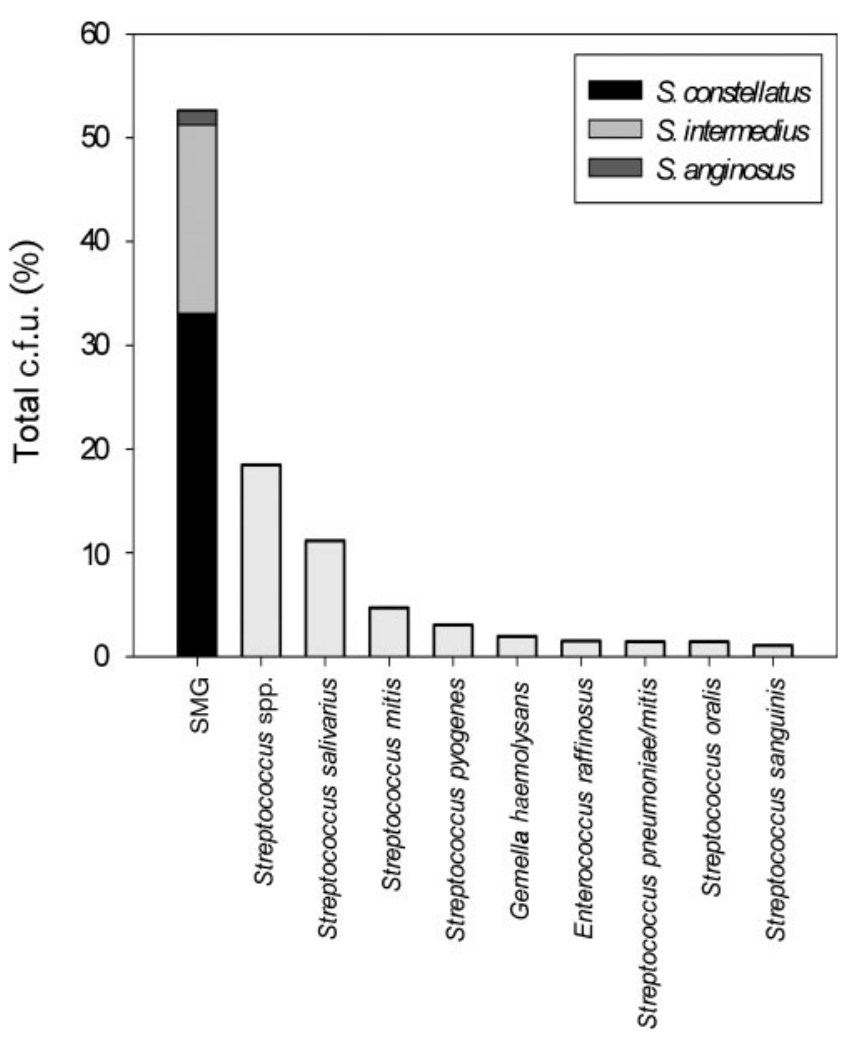

Fig. 2. The most abundant organisms cultivated on McKay agar as represented by total c.f.u. Each organism is represented as a percentage of the total c.f.u. recovered from all McKay agar plates during the 6-month study.

(five), Rothia (three), Actinomyces (two) and Lactobacillus (one).

Of the 457 viridans group streptococci, $322(70.5 \%)$ had the capacity to change the colour of the $\mathrm{pH}$ indicator in McKay agar (bromocresol purple) from purple to yellow (Table 1). Therefore, the colour change was not specific to the SMG. However, as $92.6 \%$ of the SMG strains collected generated an acidic environment on McKay agar (Table 1), the colour change is a valuable aid for distinguishing the SMG.

McKay agar cannot be used without a confirmatory test to verify the identity of putative SMG isolates because of its semi-selective nature and the complexity of the microbial communities in CF airways. As with most selective media, it was not impermeable to infrequent breakthrough growth of a small number of routine CF pathogens such as Staphylococcus aureus and other members of the oropharyngeal flora (OF). Sensitive, rapid and SMG-specific molecular tests, as alternatives to $16 \mathrm{~S}$ rRNA gene sequencing, are required because clinical laboratories are unlikely to be able to adopt a sequencing strategy for routine microbial identification. Recently, a novel biochemical scheme for SMG speciation has been developed (Grinwis 
et al., 2010); such approaches in combination with McKay agar culture could be very useful for SMG diagnostics.

\section{Correlation between high SMG loads and exacerbation infections}

We were able to determine the absolute and relative abundance of the SMG relative to other OF. The OF population comprises $\alpha$ - and $\gamma$-haemolytic isolates detected by CBA culture but considered to be clinically irrelevant. It is generally assumed that the SMG would be represented in this measure of the OF; however, this was clearly not the case. The data summarizing the relationship between the SMG and total OF concentrations are presented in Fig. 3.

The SMG concentration outnumbered the enumerated OF by at least one order of magnitude in $14(18.7 \%)$ of the SMG-positive sputum samples. In some cases, McKay agar increased the recovery of the SMG by 1000 -fold. The SMG concentration was measured below the total OF concentrations (by at least one order of magnitude) in 42 sputum samples (56\%). This highlights the increased sensitivity of McKay agar for reliably lowering the detection limit of the SMG by at least two orders of magnitude.

Sputum samples that yielded the SMG at greater than $10^{7}$ c.f.u. $\mathrm{ml}^{-1}$ were only collected at the time of admission to hospital for an acute pulmonary exacerbation (Fig. 3). Streptococcus constellatus and Streptococcus intermedius represented the numerically dominant pathogen at the

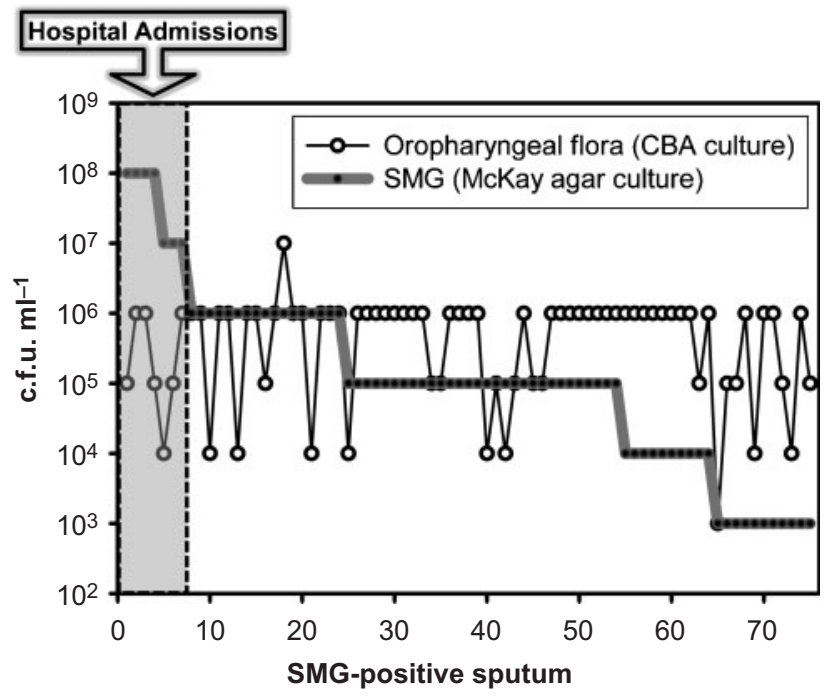

Fig. 3. The relationship between the SMG (McKay agar culture) and total OF concentration (detected by CBA culture) reveals that high SMG loads are associated with pulmonary exacerbations. The c.f.u. $\mathrm{ml}^{-1}$ of each SMG-positive culture is plotted, along with the corresponding CBA count of OF. The samples are plotted in order of highest to lowest SMG abundance. Sputum samples that were collected at the time of admission to hospital for pulmonary exacerbation are shown within the shaded box. time of hospital admission five and three times, respectively. Members of the SMG were never recovered at concentrations greater than $10^{7}$ c.f.u. $\mathrm{ml}^{-1}$ from patients who were asymptomatic. In all cases where SMG strains were detected as the numerically dominant pathogen at the onset of an acute pulmonary exacerbation, there was no evidence of an increase in the load of other principal pathogens ( $P$. aeruginosa or Staphylococcus aureus) prior to hospitalization.

Culture-independent approaches have revealed that CF airway microbiology is much more complex than the traditionally held view of chronic colonization by a few key pathogens (Bittar et al., 2008; Harris et al., 2007; Sibley et al., 2009; Tunney et al., 2008). This study highlights the utility of employing alternative cultivation-dependent practices for exploring the $\mathrm{CF}$ microbiome for unrecognized pathogens.

McKay agar culture can be a valuable addition to CF microbiology protocols in that it allows suppression of routine CF pathogens and $60-80 \%$ of viridans streptococci (with the exception of the SMG) (Brogan et al., 1997; Cooke \& O'Neill, 1998), while allowing distinct subpopulations within the OF such as the SMG to be followed over time. Using conventional techniques (CBA culture) alone, we failed to recover SMG strains when culturing polymicrobial specimens due to the apparent overgrowth of other members of the microbial community and, more frequently, because of poor recovery on CBA.

This study revealed that the SMG is common in adult CF airways, with a prevalence of greater than $40 \%$. Whilst there was considerable variation in SMG abundance among those individuals whose sputum was colonized by the SMG, certain patterns were observed. The presence of the SMG at concentrations greater than $10^{7}$ c.f.u. $\mathrm{ml}^{-1}$ was associated with symptomatic deterioration in clinical status. Furthermore, we have shown previously that a reduction in SMG bacterial loads correlates with clinical resolution of symptoms, unlike traditional CF pathogens, which may or may not demonstrate a bacteriological response (Parkins et al., 2008a; Sibley et al., 2008b).

It is important to consider that CF patients are surviving longer than ever before because of multifaceted clinical interventions. Hence, the microbiological nature of chronic airway infections may be changing in CF. It will be very interesting to determine whether the SMG carriage rates in $\mathrm{CF}$ increase with age, as they do in the general population. It will also be of interest to investigate the prevalence of the SMG in other respiratory infections. McKay agar allows routine quantitative culture of the SMG from clinical samples and would be a valuable addition to standard culture media to evaluate airway infections.

\section{ACKNOWLEDGEMENTS}

We acknowledge the Southern Alberta CF Clinic for their contribution to patient care and participation in sputum sample gathering 
and Tracie Lloyd for her efforts to coordinate specimen processing. This study was supported by a grant from Calgary Laboratory Services and The Canadian Cystic Fibrosis Foundation. M. G. S. is supported as an Alberta Heritage Foundation for Medical Research Scientist and Canada Research Chair in Microbial Gene Expression. An Alberta Heritage Foundation for Medical Research studentship and a Canada Graduate Scholarship from Canadian Institutes of Health Research support C.D.S.

\section{REFERENCES}

Bittar, F., Richet, H., Dubus, J. C., Reynaud-Gaubert, M., Stremler, N., Sarles, J., Raoult, D. \& Rolain, J. M. (2008). Molecular detection of multiple emerging pathogens in sputa from cystic fibrosis patients. PLoS One 3, e2908.

Brogan, O., Malone, J., Fox, C. \& Whyte, A. S. (1997). Lancefield grouping and smell of caramel for presumptive identification and assessment of pathogenicity in the Streptococcus milleri group. J Clin Pathol 50, 332-335.

Cooke, R. P. \& O'Neill, W. A. (1998). Laboratory identification of Streptococcus milleri. J Clin Pathol 51, 174.

Daley, P., Church, D. L., Gregson, D. B. \& Elsayed, S. (2005). Specieslevel molecular identification of invasive "Streptococcus milleri" group clinical isolates by nucleic acid sequencing in a centralized regional microbiology laboratory. J Clin Microbiol 43, 2987-2988.

Gilligan, P. H. (1991). Microbiology of airway disease in patients with cystic fibrosis. Clin Microbiol Rev 4, 35-51.

Grinwis, M. E., Sibley, C. D., Parkins, M. D., Eshaghurshan, C. S. Rabin, H. R. \& Surette, M. G. (2010). Characterization of Streptococcus milleri group isolates from expectorated sputum of adult cystic fibrosis patients. J Clin Microbiol 48, 395-401.

Harris, J. K., De Groote, M. A., Sagel, S. D., Zemanick, E. T., Kapsner, R., Penvari, C., Kaess, H., Deterding, R. R., Accurso, F. J. \& Pace, N. R. (2007). Molecular identification of bacteria in bronchoalveolar lavage fluid from children with cystic fibrosis. Proc Natl Acad Sci U S A 104, 20529-20533.

Ishida, T., Hashimoto, T., Arita, M., Ito, I. \& Osawa, M. (1998), Etiology of community-acquired pneumonia in hospitalized patients: a 3-year prospective study in Japan. Chest 114, 1588-1593.

Laupland, K. B., Ross, T., Church, D. L. \& Gregson, D. B. (2006). Population-based surveillance of invasive pyogenic streptococcal infection in a large Canadian region. Clin Microbiol Infect 12, 224-230.

Liu, W. T., Marsh, T. L., Cheng, H. \& Forney, L. J. (1997). Characterization of microbial diversity by determining terminal restriction fragment length polymorphisms of genes encoding $16 \mathrm{~S}$ rRNA. Appl Environ Microbiol 63, 4516-4522.

Lyczak, J. B., Cannon, C. L. \& Pier, G. B. (2002). Lung infections associated with cystic fibrosis. Clin Microbiol Rev 15, 194-222.

Maskell, N. A., Davies, C. W., Nunn, A. J., Hedley, E. L., Gleeson, F. V., Miller, R., Gabe, R., Rees, G. L., Peto, T. E. \& other authors (2005). U.K. controlled trial of intrapleural streptokinase for pleural infection. N Engl J Med 352, 865-874.

Miller, M. B. \& Gilligan, P. H. (2003). Laboratory aspects of management of chronic pulmonary infections in patients with cystic fibrosis. J Clin Microbiol 41, 4009-4015.

Moore-Gillon, J. C., Eykyn, S. J. \& Phillips, I. (1981). Microbiology of pyogenic liver abscess. Br Med J (Clin Res Ed) 283, 819-821.
Parkins, M. D., Sibley, C. D., Surette, M. G. \& Rabin, H. R. (2008a) The Streptococcus milleri group - an unrecognized cause of disease in cystic fibrosis: a case series and literature review. Pediatr Pulmonol 43, 490-497.

Parkins, M. D., Sibley, C. D., Surette, M. G., Gregson, D. B. \& Rabin, H. R. (2008b). Streptococcus milleri endobronchial colonization is associated with an increased rate of pulmonary disease progression in cystic fibrosis. Pediatr Pulmonol 43 (Suppl. S31), S339.

Poole, P. M. \& Wilson, G. (1979). Occurrence and cultural features of Streptococcus milleri in various body sites. J Clin Pathol 32, 764-768.

Prasad, K. N., Mishra, A. M., Gupta, D., Husain, N., Husain, M. \& Gupta, R. K. (2006). Analysis of microbial etiology and mortality in patients with brain abscess. J Infect 53, 221-227.

Rogers, G. B., Hart, C. A., Mason, J. R., Hughes, M., Walshaw, M. J. \& Bruce, K. D. (2003). Bacterial diversity in cases of lung infection in cystic fibrosis patients: $16 \mathrm{~S}$ ribosomal DNA (rDNA) length heterogeneity PCR and $16 \mathrm{~S}$ rDNA terminal restriction fragment length polymorphism profiling. J Clin Microbiol 41, 3548-3558.

Rogers, G. B., Carroll, M. P., Serisier, D. J., Hockey, P. M., Jones, G. \& Bruce, K. D. (2004). Characterization of bacterial community diversity in cystic fibrosis lung infections by use of $16 \mathrm{~S}$ ribosomal DNA terminal restriction fragment length polymorphism profiling. J Clin Microbiol 42, 5176-5183.

Rogers, G. B., Carroll, M. P., Serisier, D. J., Hockey, P. M., Kehagia, V., Jones, G. R. \& Bruce, K. D. (2005). Bacterial activity in cystic fibrosis lung infections. Respir Res 6, 49.

Rogers, G. B., Carroll, M. P., Serisier, D. J., Hockey, P. M., Jones, G., Kehagia, V., Connett, G. J. \& Bruce, K. D. (2006). Use of $16 \mathrm{~S}$ rRNA gene profiling by terminal restriction fragment length polymorphism analysis to compare bacterial communities in sputum and mouthwash samples from patients with cystic fibrosis. J Clin Microbiol 44, 2601-2604.

Shinzato, T. \& Saito, A. (1995). The Streptococcus milleri group as a cause of pulmonary infections. Clin Infect Dis 21, S238-S243.

Sibley, C. D., Rabin, H. \& Surette, M. G. (2006). Cystic fibrosis: a polymicrobial infectious disease. Future Microbiol 1, 53-61.

Sibley, C. D., Duan, K., Fischer, C., Parkins, M. D., Storey, D. G., Rabin, H. R. \& Surette, M. G. (2008a). Discerning the complexity of community interactions using a Drosophila model of polymicrobial infections. PLoS Pathog 4, e1000184.

Sibley, C. D., Parkins, M. D., Rabin, H. R., Duan, K., Norgaard, J. C. \& Surette, M. G. (2008b). A polymicrobial perspective of pulmonary infections exposes an enigmatic pathogen in cystic fibrosis patients. Proc Natl Acad Sci U S A 105, 15070-15075.

Sibley, C. D., Parkins, M. D., Rabin, H. R. \& Surette, M. G. (2009). The relevance of the polymicrobial nature of airway infection in the acute and chronic management of patients with cystic fibrosis. Curr Opin Investig Drugs 10, 787-794.

Tunney, M. M., Field, T. R., Moriarty, T. F., Patrick, S., Doering, G., Muhlebach, M. S., Wolfgang, M. C., Boucher, R., Gilpin, D. F. \& other authors (2008). Detection of anaerobic bacteria in high numbers in sputum from patients with cystic fibrosis. Am J Respir Crit Care Med 177, 995-1001.

Whiley, R. A., Beighton, D., Winstanley, T. G., Fraser, H. Y. \& Hardie, J. M. (1992). Streptococcus intermedius, Streptococcus constellatus, and Streptococcus anginosus (the Streptococcus milleri group): association with different body sites and clinical infections. J Clin Microbiol 30, 243244 